\title{
Purposive Sample Consensus: A Paradigm for Model Fitting with Application to Visual Odometry
}

\author{
Jianguo Wang and Xiang Luo
}

\begin{abstract}
RANSAC (random sample consensus) is a robust algorithm for model fitting and outliers' removal, however, it is neither efficient nor reliable enough to meet the requirement of many applications where time and precision is critical. Various algorithms have been developed to improve its performance for model fitting.

A new algorithm named PURSAC (purposive sample consensus) is introduced in this paper, which has three major steps to address the limitations of RANSAC and its variants. Firstly, instead of assuming all the samples have a same probability to be inliers, PURSAC seeks their differences and purposively selects sample sets. Secondly, as sampling noise always exists; the selection is also according to the sensitivity analysis of a model against the noise. The final step is to apply a local optimization for further improving its model fitting performance. Tests show that PURSAC can achieve very high model fitting certainty with a small number of iterations.

Two cases are investigated for PURSAC implementation. It is applied to line fitting to explain its principles, and then to feature based visual odometry, which requires efficient, robust and precise model fitting. Experimental results demonstrate that PURSAC improves the accuracy and efficiency of fundamental matrix estimation dramatically, resulting in a precise and fast visual odometry.
\end{abstract}

Keywords- robust model fitting; visual odometry; samples' reliability; samples' geometry; sampling noise

\section{Introduction}

Introduced in 1981, RANSAC is a popular algorithm for a variety of robust model fitting problems - particularly in computer vision for recovering epipolar geometry and 3D motion estimation [2]. It estimates a model that fits the provided data, while simultaneously classifies the data into inliers (samples consistent with the relation) and outliers (samples not consistent with the relation). It is a simple yet powerful technique that can estimate a model using data contaminated by a large fraction of outliers. RANSAC can be briefly summarized as a hypothesize-and-verify framework: a minimal subset of samples for model fitting is randomly selected from the entire dataset. The subset is then used to fit model hypotheses which are evaluated on the entire dataset by computing the distance of all other samples to this model and constructing an inliers' set with a threshold. This hypothesize-and-verify loop is repeated until the probability of finding a model with better consensus than the current best model falls below a predefined threshold. Then all the inliers are used for model parameter estimation [1],[4]. 
For generality and simplicity, RANSAC is based on a set of assumptions which are not true in many real situations. This leaves large room for improvement. In this paper we analyse potentials for improvement, and propose a purposive sampling algorithm named PURSAC (purposive sample consensus) to substitute random sampling. Comparing to RANSAC and MLESAC (maximum likelihood estimation sample consensus), PURSAC can detect more inliers with much fewer number of iterations and in turn can improve both the efficiency and reliability of model fitting. This is very important for applications where speed and precision is critical, such as visual odometry (VO). With analysis about a model's sensitivity against sampling noise (MSASN) and the pattern of samples' validity, PURSAC is designed to efficiently handle both sampling noise and outliers for model fitting.

The rest of the paper is organized as follows: Section II gives a detailed review of RANSAC and various approaches for its improvement at different aspects, and then introduces the principles of the proposed PURSAC. Section III describes a scheme for line fitting to justify the methodology of PURSAC. Section IV explains the details of applying PURSAC to VO, especially a method for purposive sample subsets selection considering both features' matching score and their geometry. The experimental results of VO on several scenarios are presented in Section V, which demonstrate the effectiveness and robustness of the proposed algorithm. Finally, the conclusion and discussions appear in the last section.

\section{RANSAC and Its Variants}

RANSAC can often find a correct solution even for seriously contaminated data; however, in order to achieve a high confidence level the required number of sample subsets increases exponentially, and associated computational cost is substantial. Many algorithms have been developed for increasing the efficiency of the basic RANSAC algorithm, some aiming to optimize the process of model verification while some others seeking to preferentially generate more useful hypotheses [3-7]. A comparative analysis of the state-of-the-art RANSAC algorithms and their categorization can be found in [1] and [4]. Here we review them from the aspects they targeted for improvement.

\subsection{RANSAC}

Assuming all the samples have same outlier possibility $\boldsymbol{\varepsilon}$, and ignoring the impact of sampling noise, RANSAC follows a random sampling paradigm. Fundamentally it is a stochastic algorithm without deterministic guarantees of finding the global maximum of the likelihood. A success rate $\boldsymbol{p}$ is the level of confidence of finding a consensus subset, which is a function of $\boldsymbol{\varepsilon}$, the number of iterations to be conducted $\boldsymbol{N}$ and the number of samples in a subset $\mathbf{s}$ [2].

$$
N=\frac{\log (1-p)}{\log \left(1-(1-\epsilon)^{s}\right)}
$$

For the sake of robustness, in many practical implementations $N$ is usually multiplied by a factor of ten, which increases much computational costs [1]. Without prior knowledge of $\boldsymbol{\varepsilon}$, commonly the implementations of RANSAC estimate $\boldsymbol{\varepsilon}$ adaptively, iteration after iteration. 
In practice, sampling always has noise and $\varepsilon$ may be different for each sample. By analysing the difference of $\varepsilon$, it has large potential for optimizing sample subsets selection and improving model fitting performance. As an example, assuming a required success rate $\boldsymbol{p}$ is $99 \%$ and a dataset with outlier rate $\boldsymbol{\varepsilon}=50 \%$, according to (1), the number of iterations $N$ is 16 for $s=2$ (line fitting), 145 and 587 for $s=5$ and 7 (visual odometry). If a special part of the dataset can be found having a lower outlier rate $\varepsilon=20 \%$, and sample subsets are selected only from this part, then $\mathrm{N}$ is just 5, 12 and 20 for $\mathrm{s}=2,5$ and 7 respectively. This leads to one of the strategies in PURSAC, which will be detailed in the line fitting example.

The following two subsections review the model fitting approaches applied in computer vision utilizing the difference of samples' $\boldsymbol{\varepsilon}$ and model's sensitivity against sampling noise (MSASN) respectively.

\subsection{Samples Reliability Analysis}

There are many cases that sampling process can provide some information about the samples' reliability and accuracy. In feature based image matching, the feature detection and matching stages produce a set of matches where each feature is only matched once, but some of the matches may be in error [21]. In addition to pair the features in two images, feature matching also gives similarity measures (matching scores). It has been found that matches with higher scores have higher reliability of being inliers [9],[21]. The scores or the ranking of the scores provide useful information for selecting reliable sample subsets and then improving the efficiency of model parameter estimation, which RANSAC does not utilize.

Several sampling consensus algorithms have been proposed considering this important information from features' matching. Pre-emptive RANSAC by Nister is powered by random sample consensus with pre-emptive scoring of the motion hypotheses and enable real-time ego-motion estimation [3]. PROSAC (Progressive Sample Consensus) developed by Chum and Matas tentatively and progressively selects samples from a set of higher ranked features to reduce the computational costs [9]. Uncertainty RANSAC [13] incorporates feature uncertainty and shows that this determines a decrease in the number of potential outliers, thus enforcing a reduction in the number of iterations. A deterministic RANSAC approach [14] also estimates the probability of a match to be correct. Tordoff and Murray [21] proposed guided sampling and consensus for motion estimation based on MLESAC. While MLESAC assumes a uniform prior for the validity of a match, the guidedsampling approach uses the quality function of a feature matching algorithm to derive the probability of matches' validity. Similar to PROSAC, guided sampling and consensus is also based on the evidence that a valid match is likely to have a higher matching score.

Above approaches often achieve significant computational savings in practice, since good hypotheses tend to be generated early on during the sampling process. However, it is observed in many cases, features with high matching scores often lie on a same spatial structure, such as a rich texture section or object in an image, and are potentially in a degenerate geometric configuration [4]. Thus, utilizing above approaches alone has the danger of selecting feature close to each other; therefor other strategies are needed to avoid the degenerate geometric configurations. 


\subsection{Sampling Noise Analysis}

For most model fitting tasks, two types of sampling errors must be considered: small errors (noise) and blunders (outlier) [15]. Even if a consensus subset is found after $N$ iterations, due to the sampling noise and degenerate configurations, the model and inliers decided by this subset may be largely wrong. The reason will be explained with a line fitting example in Fig.1.

There are several algorithms have been proposed to address sampling noise with different strategies. GroupSAC [6] proposed by Kai aims to handle sample subset degenerate configuration. It performs well in the cases of high outlier ratios by image segmentation for group sampling; however, it is inefficient as increasing the computational cost. Assuming inlier samples with Gaussian noise, MLESAC adopts the same sampling strategy as RANSAC to generate putative solutions, but chooses the solution to maximize the likelihood rather than the number of inliers [8]. MLESAC is a generalization of RANSAC. Capel [10] proposed a statistical bail-out test for RANSAC that permits the scoring process be terminated early and achieve computational savings. Tordoff and Murray [21] also mentioned spatial grouping of samples as a cue for further speed the search.

Chum et al. define a locally optimized RANSAC variant to deal with sampling noise and to purposively select sample subset [22]. Observing that a good model tends to find a significant fraction of the inliers, an inner RANSAC strategy is devised using only the set of inliers to the current best model. The inner RANSAC technique has the effect of improving sample consensus more rapidly than standard RANSAC, which causes the termination criterion (1) to be met earlier.

Above methods can make improvement from RANSAC in some aspect, but the improvement is usually limited and unstable. Further improvement can be achieved by strategically fusing a group of selected strategies together.

\subsection{PURSAC}

By analysing the principle and effectiveness of various approaches, we design a new algorithm PURSAC, aiming to induce better model fitting results with a smaller number of iterations. It takes three major steps to address the limitations of RANSAC and it variants.

a) Instead of assuming all the samples have same probability to be inliers, PURSAC seeks their difference and purposively selects sample sets.

b) As sampling noise always exists, PURSAC purposely selects the subsets according to the analysis of a model's sensitivity against the sampling noise, causing a selective geometric consideration in VO and line fitting.

c) The final step is to apply local optimization algorithm iteratively with all the inliers so as to further improve model fitting performance.

PURSAC can achieve results close to optimal theoretical estimation. Being a qualitative guidance in theory, PURSAC's implementation needs a quantitative analysis to design executable rules for purposive sample consensus. Two examples will be investigated. Line fitting is used as an example to describe the scheme of 
PURSAC and to justify its methodology. Then it is applied to feature based VO, to conform to its prominent requirement of efficient, robust and precise model fitting.

\section{PURSAC for Line Fitting}

Let us investigate the line fitting example in the original RANSAC paper [2]. One of the assumptions inherent in the standard termination criterion (1) is that a model computed from an uncontaminated sample subset is consistent with all inliers. In practice, this is often not the case, particularly when the data points are noisy. As showing in Fig.1, two types of sampling errors (noise and outlier) exist within the sample points. Due to sampling noise, model hypotheses selected by RANSAC with limited number of iterations usually do not fit a model well, as Line 1 in the figure. By randomly selecting a set of samples (two points for line fitting) from all the samples without considering MSASN, RANSAC likely misses some inliers and consequentially reduces the accuracy of model fitting. Original RANSAC is only effective in removing outliers but is inadequate of handling sampling noise.

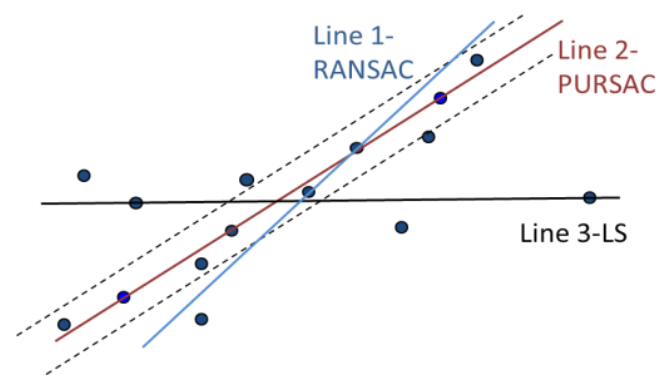

Figure 1. Line fitting results by RANSAC (Line 1), PURSAC (Line 2) and least square (Line 3).

\section{Observation1: Samples Geometry}

Two-point form of a linear equation is expressed as (2), where $\left(x_{1}, y_{1}\right)$ and $\left(x_{2}, y_{2}\right)$ are the coordinates of the two points that decide the line model.

$$
y-y_{1}=\frac{y_{2}-y_{1}}{x_{2}-x_{1}}\left(x-x_{1}\right)
$$

If the noise terms of the two points position are denoted as $\left(\delta \mathrm{x}_{1}, \delta \mathrm{y}_{1}\right)$ and $\left(\delta \mathrm{x}_{2}\right.$, $\left.\delta \mathrm{y}_{2}\right)$, and let $\mathrm{y}_{2}-\mathrm{y}_{1}$ written as $\mathrm{d} y, \mathrm{x}_{2}-\mathrm{x}_{1}$ as $\mathrm{dx}, \delta \mathrm{y}_{2}-\delta \mathrm{y}_{1}$ as $\delta \mathrm{y}$ and $\delta \mathrm{x}_{2}-\delta \mathrm{x}_{1}$ as $\delta \mathrm{x}$, Then the slope of the line can be expressed as

$$
\begin{aligned}
\left(y_{2}-y_{1}\right) /\left(x_{2}-x_{1}\right) & =\left(y_{2}-y_{1}+\delta y_{2}-\delta y_{1}\right) /\left(x_{2}-x_{1}+\delta x_{2}-\delta x_{1}\right) \\
& =(\mathrm{d} y+\delta y) /(\mathrm{d} x+\delta x) \\
& =\frac{\mathrm{d} y}{\mathrm{~d} x} \cdot \frac{1+\delta y / \mathrm{d} y}{1+\delta x / \mathrm{d} x}
\end{aligned}
$$

The items $\delta x$ and $\delta y$ are solely decided by sampling noise, while $\mathrm{d} x$ and $\mathrm{d} y$ are directly related to the distance of the two points. It can be concluded from (3) that the smaller the distance of two points $(\mathrm{d} x, \mathrm{~d} y)$ is, the more the estimated line slope to be affected by sampling noise $(\delta x, \delta y)$. This can be evidenced by the results of a Monte Carlo line fitting test. 

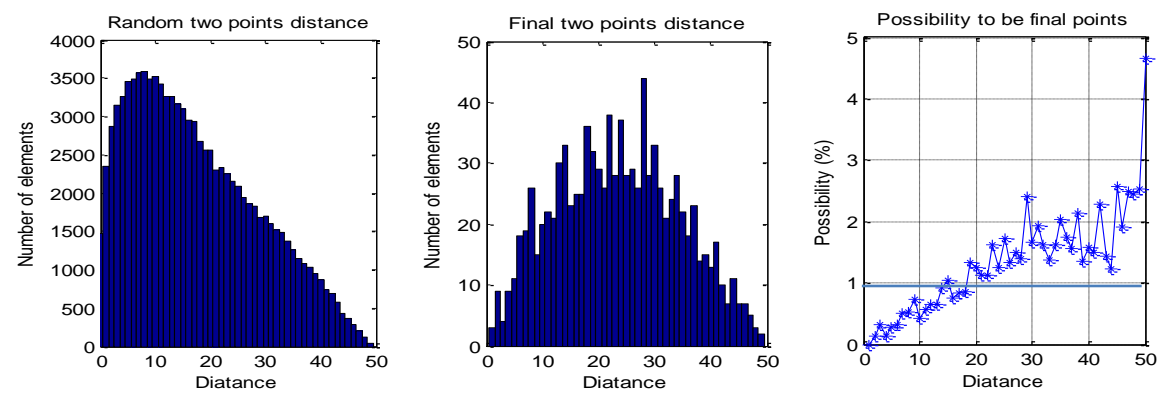

Figure 2. Monte Carlo test of the distsnce distribution for line fitting with RANSAC.

Fig. 2 shows the results of 1,000 runs' line fitting tests to verify the possibility distribution of two final points with the best model fitting against their distance. The two points are randomly selected from 100 points, so the average possibility of a point to be selected as the second point is about $1 \%$. However, as shown in the right figure, the possibility of two final points with the best model fitting increases dramatically with their distance, from almost $0 \%$ for two close points to about $3 \%$ for two far apart points. Therefore it is wise to purposely select two sample points apart, instead of random selection, so as to dilute the effect of sampling noise for model fitting.

The left figure shows that the distance between two random selected points tends to be close; while the middle figure indicates that the distance of the final two points selected by RANSAC has a Gaussian distribution with the peak at middle. The difference of these two distributions causes the result in right figure.

\section{Observation2: Samples Validity}

The sample points themselves do not have any information available about their validity for line fitting. It is impossible to rank or score the points without any prior knowledge about them. However, we do know for a line model some of the points are inliers and other are outliers, and their validity will be assessed during the process of classifying them. This validity information can be used to purposively select points afterward that have higher possibility to be inliers so as to speed up the model fitting process.

Similar to locally optimized RANSAC [22], by observing that a good model tends to find a significant fraction of the inliers, a strategic inner iteration is used. After a very small number of iterations $\boldsymbol{k}$, the inliers of the current best model are generated with RANSAC or MLESAC. Then next sample subset is selected only form the current inliers but verified against the entire dataset. As the sampling is running on only inliers, the size of sample subset can be all the inliers. This can mitigate sampling noise and minimize the error of model fitting.

This optimization technique has the effect of improving the consensus score more rapidly and causes the iteration termination criterion $(N$ in (1)) to be met earlier by selecting samples from current inliers set, which has a lower outlier rate than the entire dataset has. In addition it can also provide more robust and precise model fitting by minimizing the error of model estimation with proper sample size. 


\section{Line Fitting with PURSAC}

Considering the two observations about samples geometry and validity for the line fitting tests, PURSAC is designed to purposely select two points far apart, instead of random selection. The first point is randomly selected; then the distances from it to all the other points are calculated. The second point is selected according to the statistical distribution shown in Fig.2. Inner iteration is then applied by selecting samples only from the current inliers until reaching iteration termination criterion (1). Finally local optimization is implemented and all the inliers are used iteratively to compute the final model parameters.

Table I shows the results of 1,000 runs line fitting tests with RANSAC and PURSAC. Within 100 points, 55 are inliers. The number of iteration $\boldsymbol{N}$ is set to 20 , which means the success rate $\boldsymbol{p}$ in (1) is $99.97 \%$. Fitting_error is a model fitting error against the ground truth measured by the area between the two lines. Inliers_miss is the number of points that should be counted as inliers but miscounted as outliers; similarly is Outliers_miss. STD and mean is the standard deviation and mean of the 1,000 runs' results.

TABLE I. LINE FITTING MONTE CARLO TEST RESULTS

\begin{tabular}{|c|c|c|c|c|}
\hline $\begin{array}{c}\text { Line fitting } \\
\text { Method }\end{array}$ & $\begin{array}{c}\text { Number of inliers } \\
\text { mean/STD }\end{array}$ & $\begin{array}{c}\text { Fitting_error } \\
\text { mean/STD }\end{array}$ & $\begin{array}{c}\text { Inliers_miss } \\
\text { mean/STD }\end{array}$ & $\begin{array}{c}\text { Outliers_miss } \\
\text { mean/STD }\end{array}$ \\
\hline PURSAC & $46.07 / 0.92$ & $117.58 / 7.22$ & $11.69 / 1.80$ & $1.76 / 1.46$ \\
\hline RANSAC & $43.67 / 3.41$ & $122.99 / 22.23$ & $14.53 / 4.19$ & $2.20 / 1.69$ \\
\hline
\end{tabular}

The result shows that under exactly the same condition, PURSAC can achieve better performance than RANSAC, with less miscounted inliers and outliers, and is closer to true model. The final line fitting performance is affected by the miscounted inliers and outliers. As shown in Table I and Fig.3, all the STDs of PURSAC are smaller than that of RANSAC, indicating that PURSAC has better reliability.
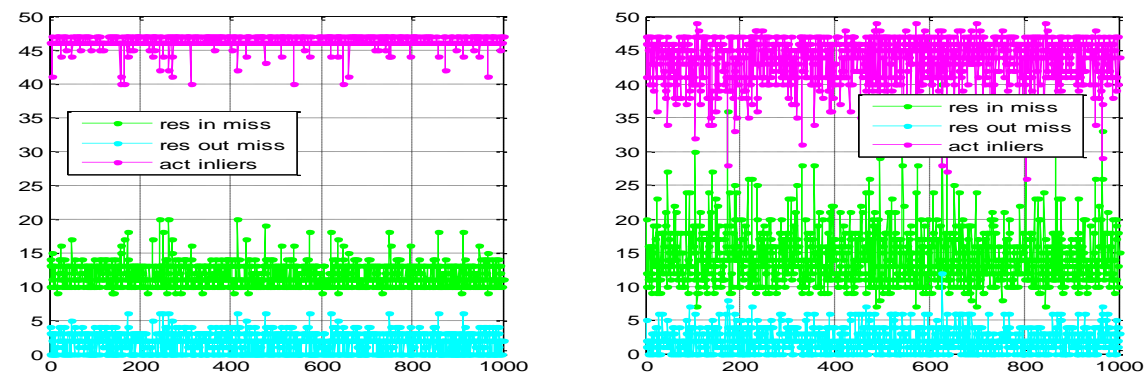

Figure 3. 1000 runs Monte Carlo test results with PURSAC and RANSAC.

The key idea behind PURSAC is to purposely select sample sets according to the sensitivity analysis of a model to be fitted against sampling noise; and also to the measures of samples' possibility to be inliers. It is worth to mention that the way to implement PURSAC is based on the analysis of each model fitting case and it is open to find an optimal way for different cases. 


\section{PURSAC for Visual Odometry}

RANSAC and MLESAC has been widely used in computer vision, particularly in the areas of recovering epipolar geometry and 3D motion estimation, such as image registration, structure from motion and feature based VO. The motion of an agent (vehicle, human or robot etc.) can be estimated by incrementally estimating the pose of the agent through examination of the movement induced on the images of its on board camera(s) [1]. Feature based VO uses salient and repeatable features extracted and matched across the captured images. No matter which algorithm is used, the matched features are usually contaminated by outliers (wrong data associations). How to efficiently remove the outliers is the most delicate task in VO and still has large room for improvement.

This section introduces how to apply PURSAC to VO. The relations of features matching scores to their possibility to be outliers and to their location accuracy in images are investigated first. Then the sensitivity analysis of the egomotion model against samples noise is conducted. Based on these analyses PURSAC is elaborated aiming to design a purposive sample set selection procedure for the fundamental matrix estimation, and to improve the results of outlier removal and model fitting and in turn the VO performance.

\section{Feature Matching Analysis}

First let us analyse the correspondence of features matching score and the features' possibility to be inliers. Similar analysis has been done previously for SIFT [16] and some other descriptors [21]. In all experiments, regardless of which similarity function used, the fraction of inliers decreased almost monotonically as a function of the number of tentative matches [9]. This is verified by our test results for 169 pairs of images using SURF features[17]. All the results show that the features with lower matching scores have higher possibility of being outliers.

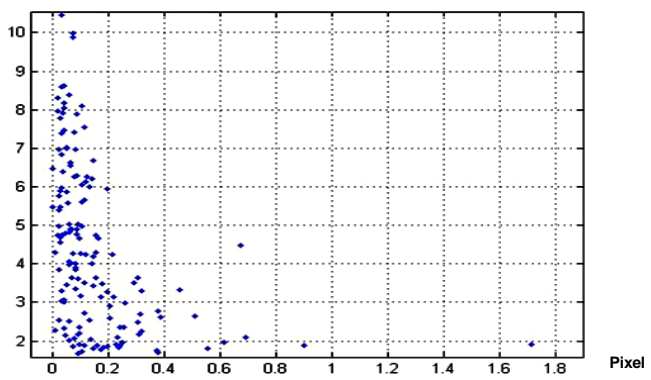

Figure 4. Features' location accuracy and matching score correspondence.

Another test was conducted to evaluate the robustness and accuracy of SIFT and SURF features' location against feature matching scores. Fig.4 is a test result for SIFT features. The horizontal axis is SIFT features' location accuracy in pixels, and vertical axis is the features' matching scores, which is the 'confidence level' of a matching is correct. It shows that as the confidence increases, so does the location accuracy (to an extent). This location error can be treated as sampling noise that is related to feature matching score. This observation will be counted in designing PURSAC rules for purposive sample subset selection. 


\section{Model Noise Sensitivity Analysis}

Model noise sensitivity analysis is to find a strategic way to dilute the effect of sampling noise for model fitting. It has been found that the geometry of selected features in images affects VO result remarkably $[10,11]$. The more evenly features are distributed in images, the less sensitive a model is to noise, and the more stable is VO results. The fact that the geometry of the features in images affects the VO results reflects the relation between sampling noise and the model to be fitted.

The fundamental matrix estimation with RANSAC etc. method takes geometric constraints introduced by a motion model. After feature detection and matching, matched features are nominated for subsequent procedure. In RANSAC, sample data sets are randomly chosen from entire matched features. However, the selected feature points may be close to each other (causing degenerate configuration), which will induce uncertainty of model fitting and affect the accuracy of estimation.

\section{PURSAC Rules for Visual Odometry}

Feature based VO requires efficient and robust model fitting. According to the statistical analysis of outlier possibility and features' location accuracy against the matching scores, rules for PURSAC implementation are innovated by considering both scores ranking and feature geometry.

a) All the matched features are ranked by their matching scores. The one with the highest rank is selected and the features close to it within a threshold $\boldsymbol{\rho}$ are excluded in following samples selection. This process iterates until all the matched features are either selected or excluded.

b) Only the selected features are used for searching the sample set for the consensus of model hypothesis but it is verified against the entire dataset. Sample subsets are purposely selected according to their ranking until reaching an initial iteration number $\boldsymbol{k}$.

c) Same as line fitting case, local optimization is then implemented to further increase the speed and certainty of model fitting. By improving accuracy and efficiency of fundamental matrix estimation, a precise and fast visual odometry can be achieved.

This set of rules is combined with both standard RANSAC and MLESAC for fundamental matrix estimation and visual odometry computation. The threshold $\rho$ is set as 10 times of the inlier threshold in RANSAC (10x1 pixels), and the initial iteration number $\boldsymbol{k}$ is set to 20 .

\section{Experiment Results}

Málaga 2009 Robotic Dataset Collection PARKING-6L is used in this paper for featured based VO testing [20]. A section of the dataset is selected from the images captured by a camera mounted on a test vehicle which runs a closed loop in a car park. The test runs 100 times to evaluate the precision and consistency of the VO results from different methods. Test results from PURSAC are compared with RANSAC and MLESAC, which were implemented in our previous approaches for VO [19] and monocular SLAM [18] respectively. 


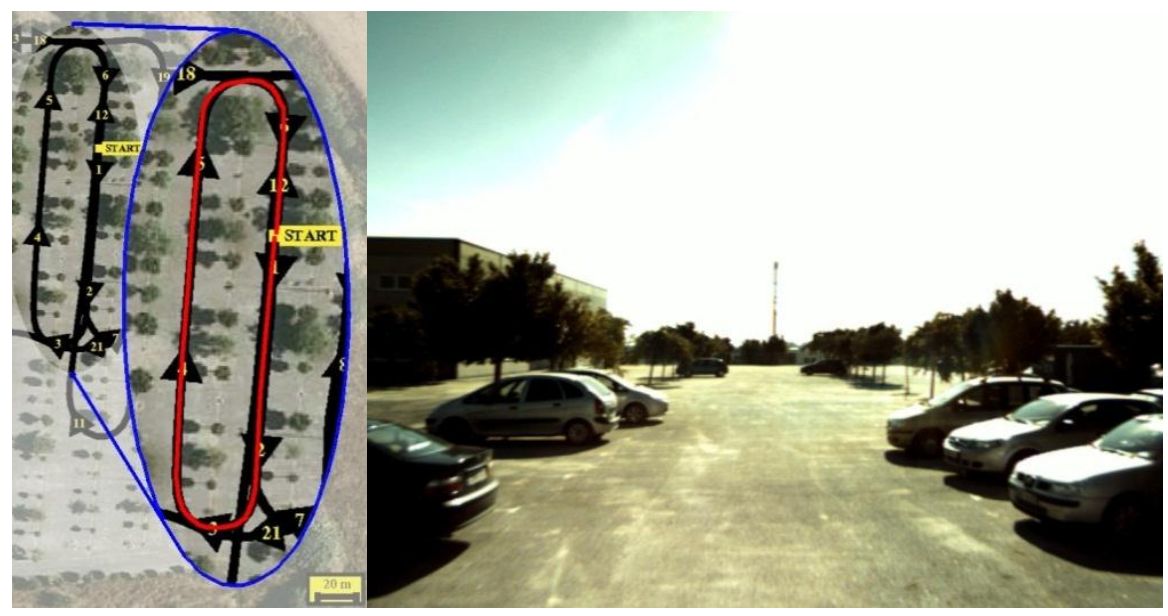

Figure 5. Test field, trajectory (left) and a sample image for visual odometry

The red line in left image of Fig. 5 is trajectory of the test in a car park. The right image is one of the images captured by an onboard camera for our visual odometry test.

Table II is the test results of matched features' inlier rate detected by different methods in 100 runs. Five pairs of images have the inlier rate from less than $50 \%$ to over $85 \%$. The tests set two different success rate $\boldsymbol{p}_{1}=99 \%$ and $\boldsymbol{p}_{2}=99.99 \%$. The number of iterations is calculated by (1) dynamically.

TABLE II. IMAGE MATCHING MONTE CARLO TEST RESULTS

\begin{tabular}{|c|c|c|c|c|c|c|c|c|c|}
\hline \multirow{2}{*}{$\begin{array}{l}\text { Image pair } \\
\text { (Number of } \\
\text { matches) }\end{array}$} & \multirow{2}{*}{$\begin{array}{c}\text { Inlier rate } \mu \\
\& \text { number of } \\
\text { iteration } N\end{array}$} & \multicolumn{2}{|c|}{$\begin{array}{c}\text { RO } \text { (RANSAC } \\
\text { Original) }\end{array}$} & \multicolumn{2}{|c|}{$\begin{array}{c}\text { RP (RANSAC } \\
\text { PURSAC) }\end{array}$} & \multicolumn{2}{|c|}{$\begin{array}{c}\text { MO (MLESAC } \\
\text { Original) }\end{array}$} & \multicolumn{2}{|c|}{$\begin{array}{c}\text { MP (MLESAC } \\
\text { PURSAC) }\end{array}$} \\
\hline & & $p_{1}$ & $p_{2}$ & $p_{1}$ & $p_{2}$ & $p_{1}$ & $p_{2}$ & $p_{1}$ & $p_{2}$ \\
\hline \multirow{3}{*}{$\begin{array}{c}\text { Image } \\
2 \& 3(357)\end{array}$} & & $68.74 \%$ & $70.03 \%$ & $75.92 \%$ & $75.83 \%$ & $68.10 \%$ & & & \\
\hline & Inlier & & & & & & & & \\
\hline & & 68.2 & & & & & & & \\
\hline \multirow{3}{*}{$\begin{array}{c}\text { Image } 27 \\
\qquad 28 \\
(390)\end{array}$} & & $52.78 \%$ & $54.32 \%$ & $\mathbf{5 8 . 3 9} \%$ & $58.46 \%$ & $52.72 \%$ & & & \\
\hline & Inliers STD & & & & & 10.23 & & & 3.1527 \\
\hline & & 447. & & & & 475 & & & 610.29 \\
\hline \multirow{3}{*}{$\begin{array}{c}\text { Image } 58 \\
\& 59 \\
(1019) \\
\end{array}$} & & $77.03 \%$ & & & & & & & \\
\hline & & 38.96 & & & & & & & \\
\hline & & 31.7 & & 2 & 39.05 & 30.89 & 8 & & 39.56 \\
\hline \multirow{3}{*}{$\begin{array}{c}\text { Image } 2 \& 4 \\
(186)\end{array}$} & & $55.22 \%$ & & & $59.70 \%$ & $55.35 \%$ & & & $59.77 \%$ \\
\hline & Inliers STD & & & & & & & & 0.6257 \\
\hline & & 335.70 & 507.45 & 230.12 & 390.37 & 346.98 & $=$ & 28.08 & 422.55 \\
\hline \multirow{3}{*}{$\begin{array}{c}\text { Image 2\&6 } \\
(129)\end{array}$} & & $44.32 \%$ & $44.99 \%$ & $47.16 \%$ & $47.22 \%$ & $43.81 \%$ & $43.95 \%$ & $47.28 \%$ & $47.38 \%$ \\
\hline & Inliers STD & 1.96 & & & 1.52 & 2.25 & 2.46 & 1.47 & 1.6161 \\
\hline & Mean $N$ & 1471.0 & \begin{tabular}{|l|}
2567.2 \\
\end{tabular} & 1127.7 & 2090.6 & 1640.6 & 3193.4 & 1338.5 & 2615.3 \\
\hline
\end{tabular}


The results show that combining with either RANSAC or MLESAC, PURSAC achieves much better results than the original algorithms. The inlier rate is higher and the standard deviation of the number of detected inliers is much lower, which means PURSAC has much better and more consistent sample consensus. It is noted that for the images with high inlier rate (Image 58\&59), the STD of both RP and MP reaches zero, entailing a complete certainty. Results show that the two different success rates $\mathrm{p}$ do not impact much on the inlier rate $\mu$ of PURSAC, but do for MLESAC and RANSAC. Even if a higher success rate $\mathrm{p} 2$ is selected for MLESAC and RANSAC, PURSAC still performs better with a low success rate, as the bold data indicated. Therefore, the number of iterations needed for PURSAC (RP and $\mathrm{MP}$ ) is much lower than the original algorithms, leading to a faster process.

The test results on 103 pair of images for 100 runs are plotted in Fig.6. The top figure shows the number of matches $N_{m}$, the number of inliers $N_{i n}$ and inlier rate $\mu$ in each pair of images. The middle one is the mean difference of $\boldsymbol{N}_{\text {in }}$ detected by MO and MP. It indicates that MP can always detect more inliers than MO, especially in the case that $N_{i n}$ is low (image number 76). The bottom figure shows the standard deviation of $\boldsymbol{N}_{\text {in }}$ for 100 runs. MO has higher STD than MP in all the 103 pair of images. This proves that MP has better consistency than original MLESAC, which is also critical for model fitting.
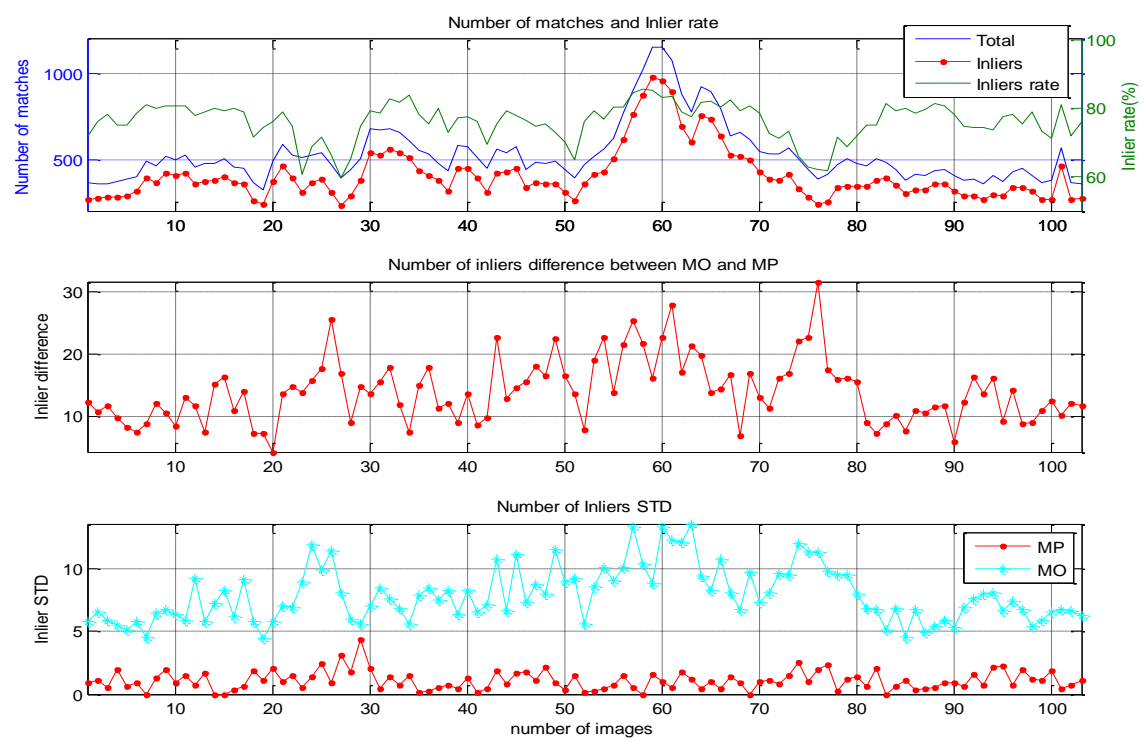

Figure 6. Number of inliers in visual odometry tests

The trajectories of 100 runs VO using MLESAC (MO) and proposed PURSAC (MP) are plotted for analysis and comparison. As shown in Fig.7, PURSAC has significantly improved the performance of $\mathrm{VO}$ in terms of standard deviation. The final position of the true trajectory returns to the start position, however, due to the camera calibration uncertainty, there is a bias in both cases. The mean and standard deviation of final camera positions with different methods are listed in Table III. 

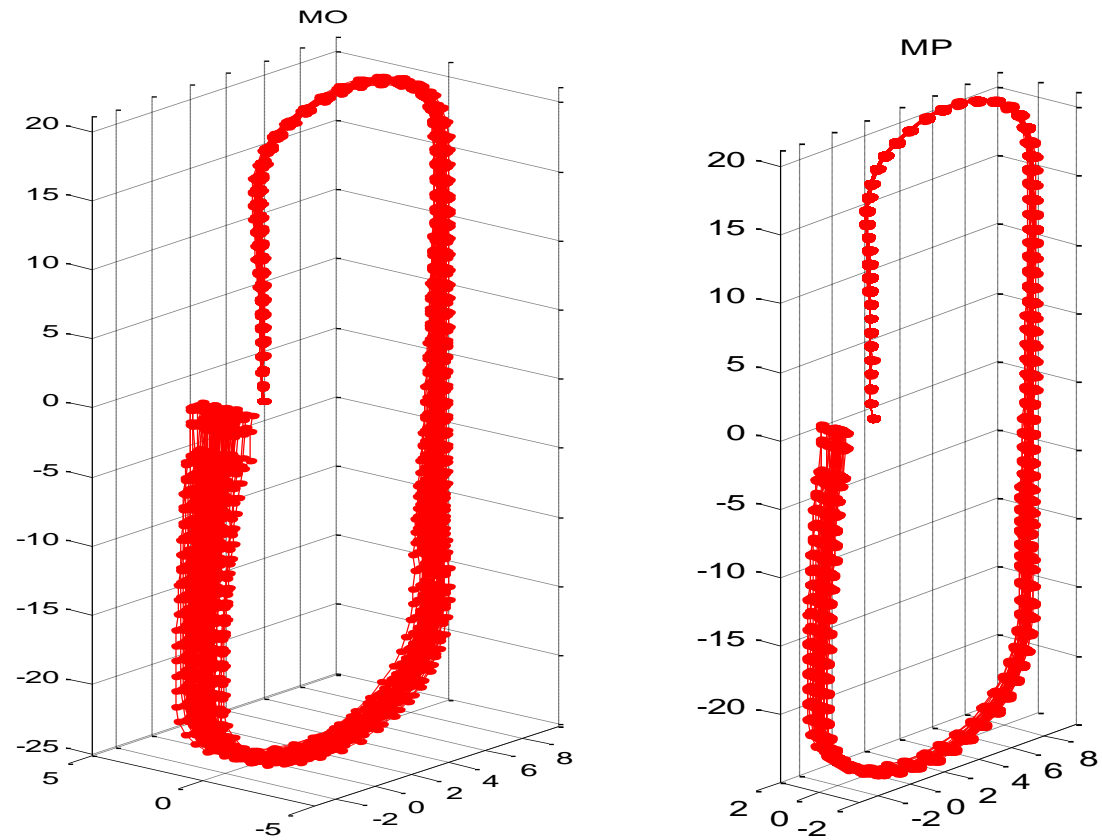

Figure 7. The 100 trajectories of $\mathrm{VO}$ using MO and MP

TABLE III. VO FINAL POSITION STD AND NUMBER OF ITERATIONS

\begin{tabular}{|l|c|l|c|c|}
\hline \multicolumn{1}{|c|}{ Methods } & X STD/mean & Y STD/mean & Z STD/mean & Mean iterations \\
\hline RO $\left(\boldsymbol{p}_{2}=99.99 \%\right)$ & $0.215 / 1.247$ & $2.220 / 1.349$ & $0.217 / 0.319$ & 147.51 \\
\hline RP $\left(\boldsymbol{p}_{\boldsymbol{I}}=99 \%\right)$ & $\mathbf{0 . 0 8 4 0} / 1.207$ & $\mathbf{0 . 2 5 2} / 0.782$ & $\mathbf{0 . 0 5 8 8} / 0.296$ & $\mathbf{6 5 . 8 7}$ \\
\hline MO $\left(\boldsymbol{p}_{2}=99.99 \%\right)$ & $0.184 / 1.208$ & $0.533 / 1.005$ & $0.151 / 0.292$ & 151.75 \\
\hline MP $\left(\boldsymbol{p}_{\boldsymbol{I}}=99 \%\right)$ & $\mathbf{0 . 0 6 2 5} / 1.204$ & $\mathbf{0 . 2 3 6} / 0.791$ & $\mathbf{0 . 0 4 1 5} / 0.295$ & $\mathbf{6 7 . 5 9}$ \\
\hline
\end{tabular}

Table III shows that the mean final camera positions with different methods are similar, which is a systemic bias to the ground truth. While for standard deviation and number of iterations, PURSAC achieves much better results than RANSAC and MLESAC. With less than half of the number of iterations, the final positions of the two PURSAC methods (RP and MP) have much smaller STD than that of the original RANSAC (RO) and MLESAC (MO).

\section{Conclusion}

This paper introduces PURSAC, a purposive sample set selection paradigm for model fitting. It has three major steps to implement. Firstly, instead of assuming all the samples have a same probability to be inliers, PURSAC seeks their differences and purposively selects sample sets. Secondly, as sampling noise always exists; the selection is also according to the sensitivity analysis of a model against the noise. The final step is to apply a local optimization for further improving its model fitting 
performance. PURSAC can combine with any model fitting algorithm that uses random sample sets selection, and achieve better outcomes with a smaller number of iterations.

Being a qualitative guidance in principle, PURSAC's implementation needs quantitative analysis to design executable rules for purposive sample consensus. Two examples are investigated in this paper. PURSAC is applied to line fitting to explain its principles, and then to visual odometry, which requires efficient, robust and precise model fitting.

Experimental results in the two examples show that PURSAC can achieve very high model fitting certainty using only a small number of iterations. It demonstrates much better performance than RANSAC and MLESAC. Applied in VO, concerning both features' geometry and matching score ranking, PURSAC improves the accuracy and efficiency of fundamental matrix estimation dramatically, resulting in a precise and fast visual odometry.

\section{References}

[1] F. Fraundorfer and D. Scaramuzza, "Visual Odometry Part II: Matching, Robustness, Optimization, and Applications," Robotics \& Automation Magazine, IEEE, vol. 19, pp. 78-90, 2012.

[2] M. A. Fischler and R. C. Bolles, "Random sample consensus: a paradigm for model fitting with applications to image analysis and automated cartography," Communications of the ACM, vol. 24, pp. 381-395, 1981.

[3] D. Nister, "Preemptive RANSAC for live structure and motion estimation," in Computer Vision, 2003. Proceedings. Ninth IEEE International Conference on, 2003, pp. 199-206 vol.1.

[4] Rahul Raguram, Jan-Michael Frahm, Marc Pollefeys, " A Comparative Analysis of RANSAC Techniques Leading to Adaptive Real-Time Random Sample Consensus", in Proceedings of the 10th European Conference on Computer Vision: Part II, pp. 500-513, 2008.

[5] J. M. Frahm and M. Pollefeys, "RANSAC for (quasi-) degenerate data (QDEGSAC)," in Computer Vision and Pattern Recognition, 2006 IEEE Computer Society Conference on, 2006, pp. 453-460.

[6] N. Kai, et al., "GroupSAC: Efficient consensus in the presence of groupings," in Computer Vision, 2009 IEEE 12th International Conference on, 2009, pp. 2193-2200.

[7] E. Rosten, et al., "Improved RANSAC performance using simple, iterative minimalset solvers," arXiv preprint arXiv:1007.1432, 2010.

[8] P. H. S. Torr and A. Zisserman, "MLESAC: A New Robust Estimator with Application to Estimating Image Geometry," Computer Vision and Image Understanding, vol. 78, pp. 138-156, 2000.

[9] O. Chum and J. Matas, "Matching with PROSAC - progressive sample consensus," in Computer Vision and Pattern Recognition, CVPR 2005. IEEE Computer Society Conference on, 2005, pp. 220-226 vol. 1. 
[10] D. Capel, "An effective bail-out test for RANSAC consensus scoring," in Proc. BMVC, 2005, pp. 629-638.

[11] D. Scaramuzza, F. Fraundorfer, and R. Siegwart, "Real-time monocular visual odometry for on-road vehicles with 1-point RANSAC," in Proc. IEEE Int. Conf. Robotics and Automation (ICRA), 2009, pp. 4293-4299.

[12] D. Nister, O. Naroditsky, and J. Bergen, "Visual odometry," in Proc. Int. Conf. Computer Vision and Pattern Recognition, 2004, pp. 652-659.

[13] R. Raguram, J. Frahm, and M. Pollefeys, "Exploiting uncertainty in random sample consensus," in Proc. ICCV, 2009, pp. 2074-2081.

[14] P. McIlroy, E. Rosten, S. Taylor, and T. Drummond, "Deterministic sample consensus with multiple match hypotheses," in Proc. British Machine Vision Conf. 2010, pp. 111.

[15] Volker Rodehorst and Olaf Hellwich, "Genetic Algorithm SAmple Consensus (GASAC) - A Parallel Strategy for Robust Parameter Estimation", in Proceedings of the 2006 Conference on Computer Vision and Pattern Recognition Workshop (CVPRW'06), 17-22 June 2006, pp. 103-111.

[16] D. Lowe, "Distinctive image features from scale-invariant keypoints," I International Journal of Computer Vision, vol. 20, no. 2, pp. 91-110, 2003.

[17] H. Bay, T. Tuytelaars, and L. V. Gool, "Surf: Speeded up robust features," in Proc. ECCV, 2006, pp. 404-417.

[18] Liang Zhao, Shoudong Huang, Lei Yan, Jack Jianguo Wang, Gibson Hu, Gamini Dissanayake, Large-Scale Monocular SLAM by Local Bundle Adjustment and Map Joining, in Proc. of the 11th. Int. Conf. Control, Automation, Robotics and Vision (ICARCV 2010), ed IEEE Technical Committee, IEEE, Singapore, December 2010, pp. 431-436.

[19] J.J. Wang, S. Kodagoda, G. Dissanayake, Vision Aided GPS/INS System for Robust Land Vehicle Navigation, Proceedings of the 22nd International Technical Meeting of The Satellite Division of the Institute of Navigation (ION GNSS 2009), Savannah, Georgia, USA, 22-25 September 2009, pp.600 - 609.

[20] Jose-Luis Blanco, Francisco-Angel Moreno, Javier Gonzalez, A Collection of Outdoor Robotic Datasets with centimeter-accuracy Ground Truth, Autonomous Robots, 2009. Volume 27, Issue 4, pp. 327-351.

[21] Ben Tordoff and David W Murray, Guided Sampling and Consensus for Motion Estimation, Computer Vision-ECCV 2002, - Springer

[22] O. Chum, J. Matas, and J. Kittler, "Locally optimized RANSAC," in Proc. DAGMSymposium, pp. 236-243, 2003. 